
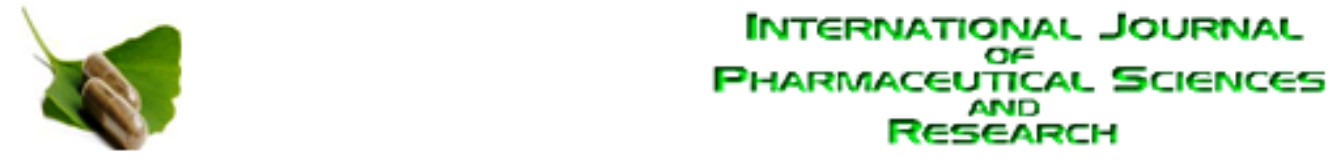

Received on 26 September, 2012; received in revised form, 03 December, 2012; accepted, 15 December, 2012

\title{
COLON TARGETED DRUG DELIVERY SYSTEM: A REVIEW
}

\section{R.B. Desi Reddy, K. Malleswari*, G. Prasad and G. Pavani}

Department of Pharmaceutics, Nalanda institute of Pharmaceutical sciences, Kantepudi, Guntur, Andhra Pradesh, India

Keywords:

Colon drug delivery, Crohn's disease, Inflammatory Bowel Disease

Correspondence to Author:

K. Malleswari

Assistant Professor, Department of Pharmaceutics, Nalanda institute of Pharmaceutical sciences, Kantepudi, Guntur, Andhra Pradesh, India

E-mail: malleswarirao24@gmail.com

\section{ABSTRACT}

Day by day there are new developments in field of colon specific drug delivery system. Colonic drug delivery has gained increased importance not just for the delivery of the drugs for the treatment of local diseases associated with the colon like Crohn's disease, ulcerative colitis, etc. but also for the systemic delivery of proteins, therapeutic peptides, anti-asthmatic drugs, antihypertensive drugs and anti-diabetic agents. New systems and technologies have been developed for colon targeting and to overcome pervious method's limitations. Colon targeting holds a great potential and still need more innovative work. This review article discusses, in brief, introduction of colon along with the novel and emerging technologies for colon targeting of a drug molecule.
INTRODUCTION: The oral aspect is considered to be most convenient for administration of drugs to Patients. Normally dissolves in stomach field as intestinal fluid and absorb from these regions of GIT. It is a serious drawback in conditions when localized delivery of drugs into the colon is required as drugs needs to be protected from the hostile environment of upper GIT.

Targeted drug delivery into the colon is highly desirable for local treatment of variety of bowl diseases such as ulcerative colitis, cirrhosis disease, amoebiasis, colonic cancer, local treatment of colonic pathologies and systemic delivery of protein and peptide drugs.

The colon specific drug delivery system (CDDS) should be capable of protecting the drug in route to the colon i.e. drug release and absorption should not occur in stomach as well as small intestine, and neither the bioactive agent should be degraded either of the dissolution sites, but only released absorbed once the system reaches the colon ${ }^{1}$.
Formulations for colonic delivery are also suitable for delivery of drugs, which are polar and/or susceptible to chemical and enzymatic degradation in upper GIT; in particular, therapeutic proteins and peptides are suitable for colonic deliveries ${ }^{2-4}$. Proteins and peptides such as insulin, calcitonin and vasopressin may be delivered systematically via colonic absorption. Other examples include novel peptides such as cytokine inhibitors and antibiotics, which are useful in treatment of IBD and GI infections respectively.

Apart from protecting these labile molecules, colon also offers an opportunistic site for oral delivery of vaccines because it is rich in lymphoid tissue. A colonic targeted approach found to be effected in minimizing uncertain side effects ${ }^{5}$.

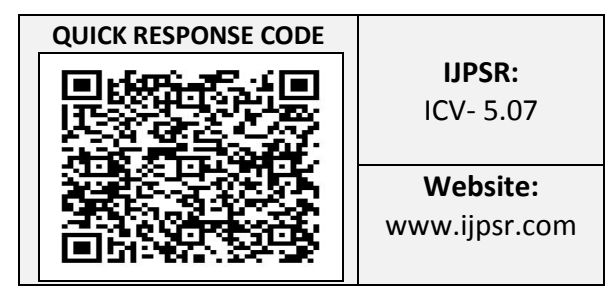


So, the colon, as a site for drug delivery, offers distinct advantages on account of near neutral $\mathrm{pH}$, a much longer transit time, relatively low proteolytic enzymatic activity and offers a much greater responsiveness to absorption enhances. Colon specific delivery systems should prevent the release of drug in upper part of GIT and require a triggering mechanism to release the drug on reaching the colon.

Why Colon Targeted Drug Delivery needed? To ensure direct treatment at the disease site, lower dosing and fewer systemic side effects. Colon-specific formulation could also be used to prolong the drug delivery. It should be considered as beneficial in the treatment of colon diseases. The colon is a site where both local or systemic drug delivery could be achieved. Topical treatment of inflammatory bowel disease, e.g. ulcerative colitis or Crohn's Disease.

Such inflammatory conditions are usually treated with glucocorticoids and Sulphasalazine. A number of others serious diseases of the colon, e.g. colorectal cancer, might also be capable of being treated more effectively if drugs were targeted to the colon. Formulations for colonic delivery are also suitable for delivery of drugs which polar and/or susceptible to chemical and enzymatic degradation in the upper Gl tract highly affected by hepatic metabolism, in particular, therapeutic proteins and peptides.

\section{Factors to be considered in the design of Colon- Specific Drug Delivery System}

1. Anatomy and Physiology of Colon: The large intestine extends from the distal end of the ileum to the anus. Human large intestine is about $1.5 \mathrm{~m}$ long ${ }^{6}$ (Table 1). The colon is upper five feet of the large intestine and mainly situated in the abdomen. The colon is a cylindrical tube that is lined by moist, soft pink lining called mucosa; the pathway is called the lumen and is approximately 2-3 inches in diameter ${ }^{7}$. The cecum forms the first part of the colon and leads to the right colon or the ascending colon (just under the liver) followed by the transverse colon, the descending colon, sigmoid colon, rectum and the anal canal (Figure 1) ${ }^{8}$. The physiology of the proximal and distal colon differs in several respects that have an effect on drug absorption at each site. The physical properties of the luminal content of the colon also change, from liquid in the cecum to semisolid in the distal colon.

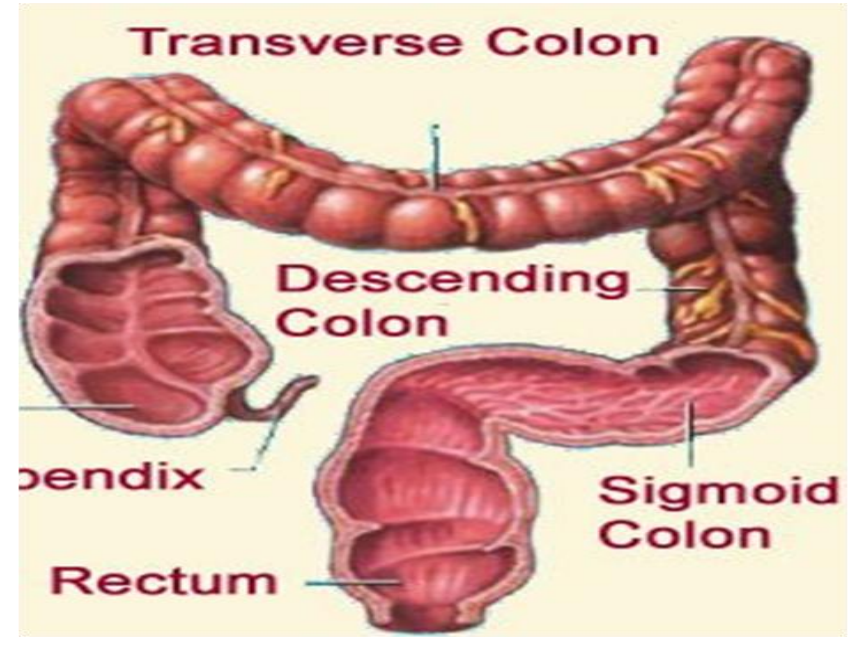

FIGURE 1: ANATOMY OF COLON

2. $\mathrm{pH}$ in the Colon: The $\mathrm{pH}$ of the gastrointestinal tract is subject to both inter and intra subject variations. Diet, diseased state and food intake influence the $\mathrm{pH}$ of the gastrointestinal fluid. The change in $\mathrm{pH}$ along the gastrointestinal tract has been used as a means for targeted colon drug delivery ${ }^{9}$. There is a $\mathrm{pH}$ gradient in the gastrointestinal tract with value ranging from 1.2 in the stomach through 6.6 in the proximal small intestine to a peak of about 7.5 in the distal small intestine (Table 1). The $\mathrm{pH}$ difference between the stomach and small intestine has historically been exploited to deliver the drug to the small intestine by way of $\mathrm{pH}$ sensitive enteric coatings. There is a fall in $\mathrm{pH}$ on the entry into the colon due to the presence of short chain fatty acids arising from bacterial fermentation of polysaccharides.

3. Transit of material in the colon: Gastric emptying of dosage forms is highly variable and depends primarily on whether the subject is fed or fasted and on the properties of the dosage form such as size and density. The arrival of an oral dosage form at the colon is determined by the rate of gastric emptying and the small intestinal transit time. The transit times of small oral dosage forms in GIT are given in Table 2. 
TABLE 1: SUMMARY OF ANATOMICAL AND PHYSIOLOGICAL FEATURES OF SMALL INTESTINE AND COLON

\begin{tabular}{|c|c|c|c|c|}
\hline \multicolumn{2}{|c|}{ Region of Gastro intestinal Tract } & \multirow{2}{*}{$\begin{array}{c}\text { Length }(\mathrm{cm}) \\
\ldots \ldots \\
\end{array}$} & \multirow{2}{*}{$\begin{array}{c}\mathbf{p H} \\
1.5-3 \text { (fasted) } \\
2-5 \text { (fed) } \\
\end{array}$} & \multirow{2}{*}{ Internal diameter $(\mathbf{c m})$} \\
\hline Stomach & & & & \\
\hline $\begin{array}{c}\text { Small } \\
\text { intestine }\end{array}$ & $\begin{array}{l}\text { Duodenum } \\
\text { Jejunum } \\
\text { lleum }\end{array}$ & $\begin{array}{c}20-30 \\
150-200 \\
200-350\end{array}$ & $\begin{array}{c}6.1 \text { (fasted),5.4(fed) } \\
5.4 \\
7-8\end{array}$ & $3-4$ \\
\hline $\begin{array}{c}\text { Large } \\
\text { intestine }\end{array}$ & $\begin{array}{c}\text { Cecum } \\
\text { Ascending colon } \\
\text { Transverse colon } \\
\text { Descending colon } \\
\text { Sigmoid colon } \\
\text { Rectum } \\
\text { Anal canal }\end{array}$ & $\begin{array}{c}6-7 \\
20 \\
45 \\
30 \\
40 \\
12 \\
3\end{array}$ & 5.5-7 & 6 \\
\hline
\end{tabular}

TABLE 2: THE TRANSIT TIME OF DOSAGE FORM IN GIT

\begin{tabular}{cc}
\hline Organ & Transit time $(\mathbf{h r})$ \\
\hline Stomach & $<1$ (Fasting) $>3($ Fed) \\
Small intestine & $3-4$ \\
Large intestine & $20-30$ \\
\hline
\end{tabular}

The movement of materials through the colon is slow and tends to be highly variable and influenced by a number of factors such as diet, dietary fiber content, mobility, stress, disease and drugs. In healthy young and adult males, dosage forms such as capsules and tablets pass through the colon in approximately 20-30 hours, although the transit time of a few hours to more than 2 days can occur. Diseases affecting colonic transit have important implications for drug delivery: diarrhea increases colonic transit and constipation decreases it. However, in most disease conditions, transit time appears to remain reasonably constant

4. Colonic Micro Flora and their enzymes: Intestinal enzymes are used to trigger drug release in various parts of the GIT. Usually, these enzymes are derived from gut micro flora residing in high number in the colon. These enzymes are used to degrade coatings/matrices as well as to break bonds between an inert carrier and an active agent (i.e., release of a drug from a prodrug. Over 400 distinct bacterial species have been found, 20-30\% of which are of the genus Bacteroides ${ }^{10,11}$. The upper region of the GIT has very small number of bacteria and predominantly consists of Grampositive facultative bacteria.
The concentration of bacteria in the human colon is 1011- $1012 \mathrm{CFU} / \mathrm{ml}$. The most important anaerobic bacteria are Bacteroides, Bifidobacterium, Eubacterium, Peptostreptococcus, peptococcus, Ruminococcus and clostridiums ${ }^{12}$. Summary of the most important metabolic reaction carried out by intestinal bacteria are given in Table 3.

Criteria for selection of drug for Colonic Drug Delivery:

1. Drug candidate: Drugs which show poor absorption from the stomach as intestine including peptide are most suitable for CDDS. The drug used in treatment of IBD, ulcerative colitis, diarrhoea and Colon cancers are ideal candidates for local colon delivery ${ }^{13}$.

2. Drug carrier The selection of carrier for particular drug candidate depends on the physiochemical nature of the drug as well as the disease for which the system is to be used. The factors such as chemical nature, stability and partition coefficient of drug and the type of absorption enhancers chosen influence the carrier selection. Moreover, the choice of drug carrier depends on the functional groups of drug molecule ${ }^{14}$. The carriers which contain additives like polymers (may be used as matrices and hydro gels as coating agents) may influence the release properties and efficacy of the systems ${ }^{15}$. 
TABLE 3: DRUG METABOLIZING ENZYMES IN THE COLON THAT CATALYZE REACTIONS

\begin{tabular}{ccc}
\hline Enzymes & Microorganism & Metabolic reaction catalyzed \\
\hline Nitroreductase & E. coli, Bacteroides & Reduce aromatic and heterocyclic nitro compounds \\
Azoreductase & Clostridia, Lactobacilli, E. coli & Reductive cleavage of azo compounds \\
Esterase and amidases & E. coli, P. vulgaris, B. subtilis, B. mycoides & Cleavage of esters or amidases of carboxylic acids \\
Glycosidase & Clostridia, Eubacterium & Cleavage of $\beta$-glycosidase of alcohols and phenols \\
Glucuronidase & E. coli, A. aerogenes & Cleavage of $\beta$-glucuronidases of alcohols and phenols \\
\hline
\end{tabular}

Advantages of CDDS over Conventional Drug Delivery:

- Chronic colitis, namely ulcerative colitis and cirrhosis disease are currently treated with glucocorticoids, and other anti-inflammatory agents

- Drugs are available directly at the target site

- Side effects can be reduced ${ }^{16}$

- Utilization of drug is more and lesser amount of dose is required comparatively ${ }^{17}$

\section{Approaches for Colonic Drug Delivery:}

\section{A. Covalent Linkage of Drug with Carrier:}

Prodrug approaches ${ }^{18}$ : Prodrug is a pharmacologically inactive derivative of a parent molecule that requires enzymatic transformation in the biological environment to release the active drug at the target site. This approach involves covalent linkage between the drug and its carrier in such a manner that upon oral administration the moiety remains intact in the stomach and small intestine, and after reached in the colon, enzymatic cleavage regenerate the drug.

Azo bond conjugate: These azo compounds are extensively metabolized by the intestinal bacteria, both by intracellular enzymatic component and extracellular reduction. The use of these azo compounds for colontargeting has been in the form of hydrogels as a coating material for coating the drug cores and as prodrug. In the latter approach the drug is attached via an azo bond to a carrier ${ }^{19}$. This azo bond is stable in the upper GIT and is cleaved in the colon by the azoreductases produced by the microflora. Sulphasalazine, used for the treatment of IBD has an azo bond between 5-ASA and sulphapyridine (SP). In the colon, the azoreductases cleave the azo bond releasing the drug, 5-ASA and the carrier SP ${ }^{19}$ (Figure 3).
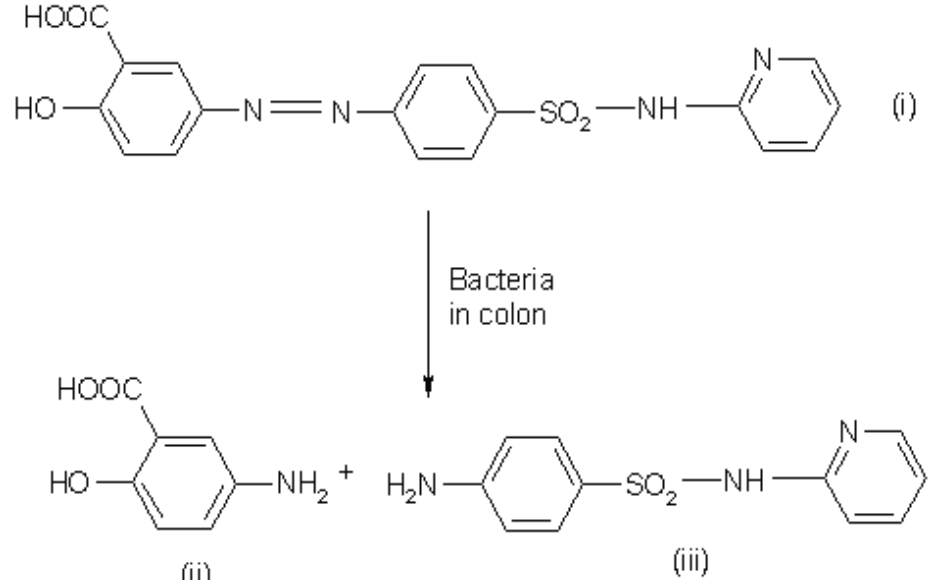

FIGURE 3. HYDROLYSIS OF SULPHASALAZINE (i) INTO 5AMINOSALICYLIC ACID (ii) \& SULFAPYRIDINE (iii)

Glycoside conjugation: Steroid glycosides and the unique glycosidase activity of the colonic microflora form the basis of a new colon targeted drug delivery system. Certain drugs can be conjugated to different sugar moieties to form glycosides. The drug part forms the aglycone and is linked to the sugar part, which forms the glycone part of the glycoside. Because they are bulky and hydrophilic, these glycosides do not penetrate the biological membranes upon ingestion. They breakdown upon action of glycosidase, releasing the drug part from the sugar. The presence of glycosidase activity in the small intestine could pose a problem in delivery of these conjugates to the large bowel, because some hydrolysis of the conjugate can be expected in the small intestine.

However, the small intestinal transit time, when compared to the large intestinal transit time, is short, and moreover, considering the time required for the hydrolysis of glycosidic bond, these conjugates can be expected to be good colon specific drug carriers. The major glycosidase enzymes produced by the intestinal microflora are $\quad \beta \quad$-D-galactosidase, $\alpha \quad-L-$ arabinofuranosidase, $\beta$-D-xylopyranosidase, and $\beta-$ Dglucosidase. These glycosidase enzymes are located at the brush border and hence are accessible to substrate easily. 
Example: lucosides, galactosides, and cellobiosides of dexamethasone, prednisolone, hydrocortisone, and fludrocortisone. Daxamethasone-21- $\beta$-glucoside, Prednisolone-21- $\beta$-glucoside.

Glucoronide conjugates ${ }^{20}$ : Bacteria of the lower GIT secrete b-glucuronidase and can deglucuronidate a variety of drugs in the intestine. Thus, the deglucuronidation process results in the release of the active drug again and enables its reabsorption. Example: Opiates, when taken for the relief of pain, cause severe constipation by inhibiting GIT motility and secretions. Narcotic antagonists, when given as antidotes for GIT side effects, immediately relieve constipation but precipitate acute withdrawal. This is because these narcotic antagonists are not selective and they not only affect the GIT activity, but also the central nervous system (CNS). A novel approach would be to target these antagonists to the lower bowel so that they are not absorbed systemically.

With this purpose, naloxone and nalmefene glucuronide prodrugs were prepared to target these drugs to the colon. When given orally to morphine dependent rats these prodrugs showed increased GIT motility and secretion in the large bowel results in a diarrhea and The resultant diarrhea flushed out the drug/prodrug from the colon thereby preventing the systemic absorption of the antagonist, which in-turn caused absence of withdrawal symptoms. Budesonideb-glucuronide prodrug also found to be superior to budesonide itself for the treatment of colitis in the rat.

Cyclodextrin Conjugate: Cyclodextrins are cyclic oligosaccharides consisted of six to eight glucose units through -1, 4 glucosidic bonds and have been utilized to improve certain properties of drugs such as solubility, stability and bioavailability. The interior of these molecules is relatively lipophilic and the exterior relatively hydrophilic, they tend to form inclusion complexes with various drug molecules. They are known to be barely capable of being hydrolyzed and only slightly absorbed in passage through the stomach and small intestine however, Colonic bacteria are capable of degrading cyclodextrins for carbon source by stimulating cyclodextranase activity. They are fermented by the colonic microflora to form small saccharides that are then absorbed.
This susceptibility to degradation specifically by colonic micro flora together with their property to form inclusion complexes with various drugs makes them particularly useful in carrying drug moieties to the colon.

The $a-$ and b-cyclodextrins are practically resistant to gastric acid, salivary, and pancreatic amylases. A clinical study has shown clear evidence that bcyclodextrin is poorly digested in the small intestine but is almost completely degraded by the colonic microflora.

Dextran Conjugate ${ }^{21}$ : Dextrans are polysaccharides of bacterial origin where the monosaccharides are joined to each other by glycoside linkages. These linkages are hydrolyzed by moulds, bacteria, and mammalian cells. The enzyme responsible for the hydrolysis of these linkages is dextranase. The dextranase activity is almost absent in the upper GIT, where as high dextranase activity is shown by anaerobic gramnegative bacteria, especially the Bacteroides, which are present in a concentration as high as 1011 per gram in colon. This led to the use of dextran as carriers for drug molecules to the colon ${ }^{22}$.

In the colon, dextran's glycosidic bonds are hydrolyzed by dextranases to give shorter prodrug oligomers, which are further split by the colonic esterases to release the drug free in the lumen of the colon. Dextran prodrug approach can be used for colonspecific delivery of drugs containing a carboxylic acid function (-COOH).NASIDS ware directly coupled to dextran by using carboxylic groups of drugs. Example is Naproxen-dextran conjugate. Glucocorticoids do not possess $-\mathrm{COOH}$ group so these are linked to dextran using spacer molecule. e.g. glucocorticoid-dextran conjugates.

Amino acid Conjugation: Due to the hydrophilic nature of polar groups like $-\mathrm{NH} 2$ and $-\mathrm{COOH}$, that is present in the proteins and their basic units (i.e. the amino acids), they reduce the membrane permeability of amino acids and proteins. Increase in hydrophilicity and chain length of carrier amino acid; decrease the permeability of amino acids and proteins. So the amino acid conjugate show more enzymatic specificity for hydrolysis by colonic enzyme ${ }^{23}$. 
Polymeric prodrugs ${ }^{24}$ : Newer approaches are aimed at use of polymers as drug carriers for drug delivery to the colon. Both synthetic as well as naturally occurring polymers are used for this purpose. Sub synthetic polymers have used to form polymeric prodrug with azo linkage between the polymer and drug moiety.

\section{B. Approaches to deliver intact molecule to colon $\mathrm{pH}$} dependent approach ${ }^{25}$ : This approach utilizes the existence of $\mathrm{pH}$ gradient in the GIT that increases progressively from the stomach $(\mathrm{pH} 1.5-3.5)$ and small intestine (5.5-6.8) to the colon (6.4-7.0).By combining the knowledge of the polymers and their solubility at different $\mathrm{pH}$ environments, delivery systems can be designed to deliver drugs at the target site. The most commonly used $\mathrm{pH}$ dependent polymers are derivatives of acrylic acid and cellulose.

\section{Coating of the Drug Core with pH sensitive Polymers} (Table 4): The intact molecule can be delivered to the colon without absorbing at the upper part of the intestine by coating of the drug molecule with the suitable polymers, which degrade only in the colon. The drug core includes tablets, capsules, pellets, granules, microparticles or nanoparticles. The coating of $\mathrm{pH}$-sensitive polymers to the tablets, capsules or pellets provide delayed release and protect the active drug from gastric fluid. The polymers used for colon targeting, however, should be able to withstand the lower $\mathrm{pH}$ values of the stomach and of the proximal part of the small intestine and also be able to disintegrate at the neutral of slightly alkaline $\mathrm{pH}$ of the terminal ileum and preferably at the ileocecal junction.

The majority of enteric and colon targeted delivery systems are based on the coating of tablets or pellets, which are filled into conventional hard gelatin capsules. The problem with this approach is that the intestinal $\mathrm{pH}$ may not be stable because it is affected by diet, disease and presence of fatty acids, carbon dioxide, and other fermentation products. Moreover, there is considerable difference in inter- and intranindividual gastrointestinal tract $\mathrm{pH}$, and this causes a major problem in reproducible drug delivery to the large intestine Eudragit-L dissolves at a $\mathrm{pH}$ level above 5.6 and is used for enteric coating, whereas Eudragit $\mathrm{S}$ is used for the colon delivery it dissolves at $\mathrm{pH}$ greater than 7.0 (attributable to the presence of higher amounts of esterified groups in relation to carboxylic groups), which results in premature drug release from the system. Problem of premature drug release can be overcome by the use of Eudragit FS.

\section{TABLE 4: VARIOUS PH DEPENDENT COATING POLYMERS}

Acidic environment of the stomach and to undergo a lag time of predetermined span of time,

\begin{tabular}{cc}
\hline Polymer & Threshold pH \\
\hline Eudragit L 100 & 6.0 \\
\hline Eudragit S 100 $^{\text {Eudragit }}{ }^{\circledR}$ L-30D & 7.0 \\
\hline Eudragit $^{\oplus}$ FS 30D & 5.6 \\
\hline $\begin{array}{c}\text { Hydroxy propyl } \\
\text { methylcellulose phthalate 50 }\end{array}$ & 6.8 \\
\hline $\begin{array}{c}\text { Hydroxy propyl methyl } \\
\text { cellulose phthalate 55 }\end{array}$ & 5.2 \\
\hline Cellulose acetate trimellate & 5.4 \\
\hline
\end{tabular}

Embedding in pH-sensitive Matrices: The drug molecules are embedded in the polymer matrix. Extrusion spheronization technique can be used to prepare uniform-size sturdy pellets for colon targeted drug delivery when it is not possible to obtain mechanically strong granules by other methods. Excipients had a significant impact on the physical characteristics of the pellets. Eudragit S100 as a pH sensitive matrix base in the pellets increased the pellet size and influenced pellet roundness. Citric acid promoted the pelletization process resulting in a narrower area distribution. However, EudragitS100 could not cause statistically significant delay in the drug release at lower $\mathrm{pH}$.

Time Dependent Delivery: It also known as pulsatile release, delayed or sigmoidal release system. This approach is based on the principle of delaying the release of the drug until it enters into the colon. Although gastric emptying tends to be highly variable, small intestinal transit time is relatively constant or little bit variation can be observed. The strategy in designing timed-released systems is to resist the release of drug take place.

The lag time in this case is the time requires to transit from the mouth to colon. A lag-time of 5 hours is usually considered sufficient since small intestine transit is about 3-4 hours, which is relatively constant and hardly affected by the nature of formulation administered. 
Time-controlled systems are useful for synchronous delivery of a drug either at pre-selected times such that patient receives the drug when needed or at a preselected site of the GI tract. These systems are therefore particularly useful in the therapy of diseases, which depend on circadian rhythms. This system has some disadvantages as follows:

- Gastric emptying time varies markedly between subjects or in a manner dependent on type and amount of food intake.

- Gastrointestinal movement, especially peristalsis or contraction in the stomach would result in change in gastrointestinal transit of the drug.

- Accelerated transit through different regions of the colon has been observed in patients with the IBD, the carcinoid syndrome and diarrhea and the ulcerative colitis.

Therefore time dependent systems are not ideal to deliver drugs to colon specifically for the treatment of colon related diseases. Appropriate integration of $\mathrm{pH}$ sensitive and time release functions into a single dosage form may improve the site specificity of drug delivery to the colon.

a) Pulsincap (Figure 4): The first formulation introduced based on this principle was Pulsincap ${ }^{\circledR}$ developed by R.R.Scherer International Corporation, Michigan, US. It consists of non disintegrating half capsule body filled with drug content sealed at the opened end with the hydrogel plug, which is covered by water soluble cap. The whole unit is coated with an enteric polymer to avoid the problem of variable gastric emptying. When the capsule enters the small intestine the enteric coating dissolves and the hydrogel plug starts to swell. The length of the plug and its point of insertion into the capsule controlled the lag time. For water-insoluble drugs, a rapid release can be ensured by inclusion of effervescent agents or disintegrants.

The plug material consists of insoluble but permeable and swellable polymers (eg, polymethacrylates), erodible compressed polymers (eg, hydroxypropylmethyl cellulose, polyvinyl alcohol, polyethylene oxide), congealed melted polymers (eg, saturated polyglycolated glycerides, glyceryl monooleate), and enzymatically controlled erodible polymer (eg, pectin).

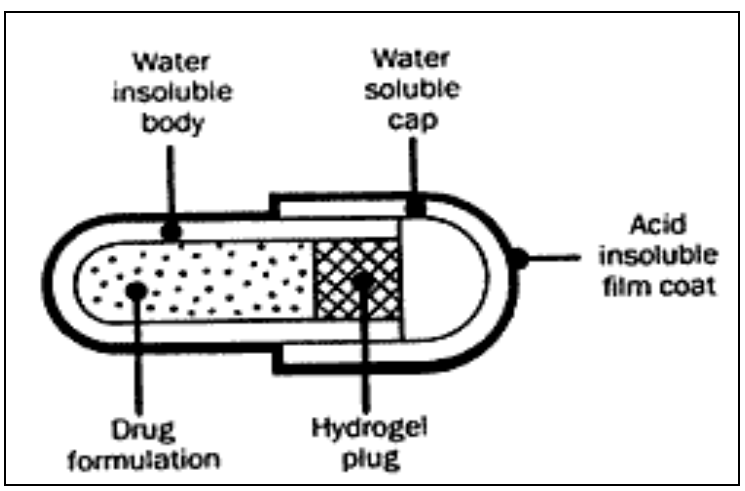

FIGURE 4: DESIGN OF PULSINCAP SYSTEM

b) Colon-Targeted Delivery Capsule based on $\mathrm{pH}$ sensitivity and Time-Release principles: The system contains an organic acid that is filled in a hard gelatin capsule as a pH-adjusting agent together with the drug substance. This capsule is then coated with a three-layered film consisting of an acid-soluble layer, a hydrophilic layer, and an enteric layer. After ingestion of the capsule, these layers prevent drug release until the environmental $\mathrm{pH}$ inside the capsule decreases by dissolution of the organic acid, upon which the enclosed drug is quickly released. Therefore, the onset time of drug release is controlled by the thickness of the acid-soluble layer.

c) Chronotropic System: The Chronotropic system consists of a drug-containing core coated by hydrophilic swellable hydroxypropylmethyl cellulose (HPMC), which is responsible for a lag phase in the onset of release. In addition, through the application of an outer gastric-resistant enteric film, the variability in gastric emptying time can be overcome, and a colon-specific release can be obtained, relying on the relative reproducibility of small intestinal transit time. The lag time is controlled by the thickness and the viscosity grades of HPMC. The system is suitable for both tablets and capsules.

d) PORT System: The Port system was developed by Therapeutic System Research Laboratory Arm Arbor, Michigan, USA, and consists of a gelatin 
capsule coated with a semi permeable membrane. Inside the capsule an insoluble plug (lipidic) consisting of osmotically active agent and the drug formulation. When in contact with the aqueous medium, water diffuses across the semi permeable membrane, resulting in increased inner pressure that ejects the plug after a lag time. The lag time is controlled by coating thickness. The system showed good correlation in lag times of in-vitro and in-vivo experiments in humans. The system proposed to deliver methylphenidate for the treatment of attention deficit hyperactivity disorder (ADHD) in school-age children.

Microbially Triggered Drug Delivery to Colon: The microflora of colon is in the range of 1011- 1012 $\mathrm{CFU} / \mathrm{ml}$. Consisting mainly of anaerobic bacteria eg: bacteroides, bifidobacteria, eubacteria, clostridia, and enterococci., enterobacteria and pnemiococcus etc., thus vast microflora fulfills its energy needs by various types of substrates that have been left undigested in small intestine ex:- di \& tri saccharides, polysaccharides etc..., ${ }^{28}$ for this fermentation the microflora, produces a vast number of enzymes like glucoridase, xylosidase, arabinosidase, galactosidase, nucleoreductase, azoreductases, deaminase and urea dehydroxylase, Because of the presence of biodegradable enzymes only in the colon, the use of biodegradable polymers for colon specific drug delivery seems to be more site specific approach as compared to other approaches.

These polymer shield the drug from the environment of stomach and small intestine and are able to deliver the drug to the colon on reacting the colon, they undergo assimilation by micro organism as degradation by enzyme as breakdown of polymer back bone leading to subsequent reduction in their molecular weight and thereby loss of mechanical strength.

Bioadhesive Systems: Oral administration of some drugs requires high local concentration in the large intestine for optimum therapeutic effects. Bioadhesion is a process by which a dosage form remains in contact with particular organ for an augmented period of time. This longer residence time of drug would have high local concentration or improved absorption characteristics in case of poorly absorbable drugs. This strategy can be applied for the formulation of colonic drug delivery systems. Various polymers including polycarbophils, polyurethanes and polyethylene oxidepolypropylene oxide copolymers have been investigated as materials for Bioadhesive systems. Bioadhesion has been proposed as a means of improving the performance and extending the mean residence time of colonic drug delivery systems.

Pressure Controlled System: The digestive processes within the GI tract involve contractile activity of the stomach and peristaltic movements for propulsion of intestinal contents. In the large intestine, the contents are moved from one part to the next, as from the ascending to the transverse colon by forcible peristaltic movements commonly termed as mass peristalsis. These strong peristaltic waves in the colon are of short duration, occurring only three to four times a day. However, they temporarily increase the luminal pressure within the colon, which forms the basis for design of pressure-controlled systems.

The luminal pressure resulting from peristaltic motion is higher in the colon compared to pressure in the small intestine, which is attributed to the difference in the viscosity of luminal contents. In the stomach and small intestine, contents are fluidic because of abundant water in digestive juices, but in the colon, the viscosity of the content is significantly increased due to reabsorption of water from the lumen and formation of feces. It has therefore been concluded that drug dissolution in the colon could present a problem in relation to colon-specific oral drug delivery systems.

Takaya et al., (1995) have developed pressure controlled colon delivery capsules prepared using an ethyl cellulose, which is insoluble in water. In such systems drug release occurs following disintegration of water insoluble polymer capsule as a result of pressure in the lumen of the colon. The thickness of the ethyl cellulose membrane is the most important factor for disintegration of the formulation. The preferred thickness of the capsule wall is about 35-60 $\mu \mathrm{m}$. The system also appeared to depend on capsule size and density. In pressure-controlled ethyl cellulose singleunit capsules the drug is in a liquid.

Lag times of three to five hours in relation to drug absorption were noted when pressure-controlled capsules were administered to human. 
Osmotic Controlled Drug Delivery: The OROS-CT system (Figure 5) can be single osmotic unit or may incorporate as many as 5-6 push-pull units, each $4 \mathrm{~mm}$ in diameter, encapsulated within a hard gelatin capsule. Each push-pull unit is bilayered laminated structure containing an osmotic push layer and a drug layer, both surrounded by a semi permeable membrane. In principle semi permeable membrane is permeable to the inward entry of water and aqueous GI fluids and is impermeable to the outward exit of the drug. An orifice is drilled into the semi permeable membrane to the drug layer.

The outside surface of the semi permeable membrane is then coated by eudragit ${ }^{\circledR} 100$ to delay the drug release from the device during its transit through the stomach. Upon arrival on the small intestine the coating dissolves at $\mathrm{pH} \leq 7$. As a result water enters the unit causing the osmotic push compartment to swell forcing the drug out of the orifice into colon. For treating ulcerative colitis, each push pull unit is designed with a 3-4 hour post gastric delay to prevent drug delivery in the small intestine. Drug release begins when the unit reaches the colon. OROS-CT units can maintain a constant release rate for up to $24 \mathrm{~h}$ in the colon

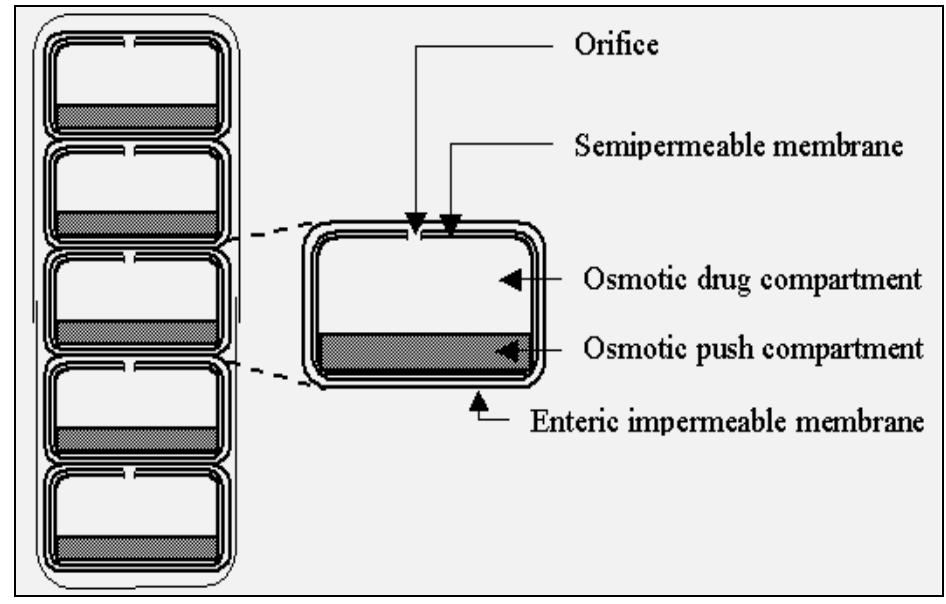

FIGURE 5: CROSS SECTION OF THE OROS-CT COLON TARGETED DRUG DELIVERY SYSTEM

Multiparticulate systems: Single unit colon targeted drug delivery system may suffer from the disadvantage of unintentional disintegration of the formulation due to manufacturing deficiency or unusual gastric physiology that may lead to drastically compromised systemic drug bioavailability or loss of local therapeutic action in the colon.
Report suggests that drug carrier systems larger than $200 \mu \mathrm{m}$ possess very low gastric transit time due to physiological condition of the bowel in colitis. And for this reason and considering the selective uptake of micron or submicron particles by cancerous and inflamed cells/tissues a Multiparticulate approach is expected to have better pharmacological effect in the colon. Recently, much emphasis is being laid on the development of multiparticulate dosage forms in comparison to single unit systems because of their potential benefits like,

- Multiparticulate systems enabled the drug to reach the colon quickly and were retained in the ascending colon for a relatively long period of time and hence increased bioavailability.

- Because of their smaller particle size as compared to single unit dosage forms these systems are capable of passing through the GI tract easily, leading to less inter- and intra subject variability.

- Moreover, Multiparticulate systems tend to be more uniformly dispersed in the Gl tract and also ensure more uniform drug absorption.

- Reduced risk of systemic toxicity, reduced risk of local irritation and predictable gastric emptying

Design of Multiparticulate Drug Delivery Systems: The purpose of designing multiparticulate dosage form is to develop a reliable formulation that has all the advantages of a single unit formulations and yet devoid of the danger of alteration in drug release profile and formulation behavior due to unit to unit variation, change in gastro luminal $\mathrm{pH}$ and enzyme population.

pH and Time Dependent Systems: One of the simplest approaches for designing $\mathrm{pH}$ dependent multiparticulate colon specific delivery system is to formulate enteric coated granules (Figure 6). Most commonly used $\mathrm{pH}$-dependent coating polymers for oral delivery are methacrylic acid copolymers, Eudragit L100 and Eudragit S100, which dissolve at pH 6.0 and 7.0 respectively. The combination of these two polymers in various ratios makes it possible to manipulate drug release within $6.0-7.0 \mathrm{pH}$ range. 
Incorporation of organic acid in both the enteric coated granules as well as the tablet matrix retarded the in vitro release and in vivo absorption of the drug because of the prolongation in disintegration time of the core system due to the presence of the acid.

In another approach, 5-fluorouracil granular matrices were designed for release of the drug in the descending colon in a controlled fashion for the treatment of colorectal carcinoma. The Glyceryl palmitostearate matrices (retardant material) coated by Eudragit S100 and were then covered by a layer of chitosan $\mathrm{HCl}$ and loaded inside HPMC capsules coated with $30 \mathrm{D}$. Upon hydration, the capsule shell dissolves and the chitosan layer forms a gel (internal pH of 4.5), which generates an acidic environment around the Eudragit film so that it does not dissolve in the ascending colon.

In the ascending colon, the chitosan $\mathrm{HCl}$ gel is degraded by the colonic micro flora, thereby exposing the Eudragit film to the colonic environment. But since the ascending colon is weakly acidic where $\mathrm{pH}$ is less than 7.0, the film coat still remains intact. However, on arrival in the descending colon where $\mathrm{pH}$ is greater than 7, the Eudragit film coat dissolves and the drug is released in a controlled fashion from the matrices. It is accepted that a colonic delivery system which is based only on GI transit time or $\mathrm{pH}$ of the GI tract would not be reliable because of the inherent variability of $\mathrm{pH}$ and emptying times from the $\mathrm{Gl}$ tract.

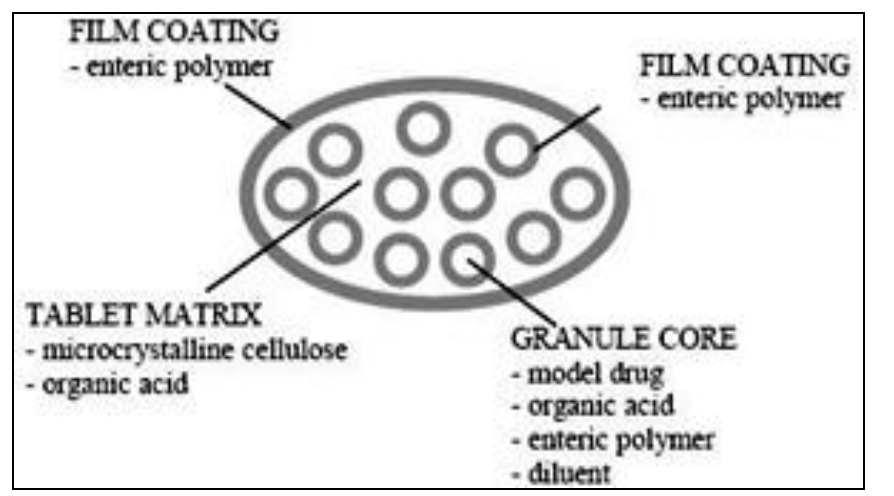

FIGURE 6: STRUCTURE OF MULTIPLE UNIT COLON SPECIFIC TABLET DEVELOPED

Micro particulate systems: In the treatment of IBD, sustained release devices like pellets, capsules or tablets have less efficiency due to diarrhea, a symptom of IBD that enhances their elimination and reduces the total time available for drug release.
It has been shown that drug carrier systems with a size larger than $200 \mu \mathrm{m}$ would be subjected to speedy bowel evacuation due to diarrhea, resulting in a decreased GI transit time and decreased efficiency. Therefore, a multiparticulate system in the micron size range could be a useful option in the design of a suitable dosage form for IBD.

Typical formulation: Eudragit P-4135 F, a new $\mathrm{pH}-$ sensitive polymer was used to prepare micro particles of tacrolimus, an immunosuppressant drug, for colonic delivery. The use of Eudragit P-4135 F in the microencapsulation of 5 -fluorouracil for the treatment of colorectal cancer has been reported. Eudragit P$4135 \mathrm{~F}$ belongs to the $\mathrm{pH}$-sensitive Eudragit group of polyacrylates and possesses a dissolution threshold $\mathrm{pH}$ slightly above 7.2. This is very useful as ulcerative colitis mainly affects the distal parts of the colon and an early drug loss towards the non-inflamed tissue would be undesirable.

Eudragit P-4135 F might prove a useful alternative for systems intended for targeting to the distal colon. It is reported that biodegradable microspheres could be efficiently taken up by macrophages. Therefore, the direct uptake of anti-inflammatory agents loaded microspheres by macrophages would have a superior immunosuppressive effect and be more useful for treatment of patients with IBD.

Micro particulates in the delivery of Peptides: The colon has always attracted attention as a potential site for the systemic absorption of peptide drugs on account of its lower proteolytic enzyme activity compared to the upper GI tract.

- Formulation 1: A system consisting of insulin encapsulated by polyacrylates wherein the coating was meant to dissolve only in the colon

- Formulation 2: A terpolymer of styrene and hydroxy ethyl methacrylate cross-linked with a difunctional azo- compound has also been reported for the delivery of insulin. The system depends on cleavage of azo bond by colonic microflora resulting in degradation of polymer and release of insulin. 
Nanoparticulate Systems: Nanoparticle size colloidal carriers composed of natural or synthetic polymers have also been investigated for colon targeting. Orally administered nanoparticles serve as carriers for different types of drugs and have been shown to enhance their solubility, permeability and bioavailability.

Nanoparticles have also been investigated for the delivery of protein and peptide drugs. For colonic pathologies, it was shown that nanoparticles tend to accumulate at the site of inflammation in IBD. This is because in case of colitis, a strong cellular immune response occurs in the inflamed regions due to increased presence of neutrophils, Natural Killer cells, macrophages and so on. It has been reported that microspheres and nanoparticles could be efficiently taken up by these macrophages. This results in accumulation of the particulate carrier system resulting in prolonged residence time in the desired area. However, an important area of concern is to prevent loss of Nanoparticle in the early transit through GI tract in order to optimize therapeutic efficacy.

Moreover, particle uptake by Payer's patches and/or enzymatic degradation may cause the release of entrapped drug leading to systemic drug absorption and side effects. In order to overcome this problem, drug loaded nanoparticles were entrapped into $\mathrm{pH}$ sensitive microspheres, which serve to deliver the incorporated nanoparticle to their site of action, thereby preventing an early drug leakage. The use of nanoparticles for bioadhesion purposes has also been investigated. Nanoparticles have a large specific surface, which is indicative of high interactive potential with biological surfaces. Since the interaction is of nonspecific nature, bioadhesion can be induced by binding nanoparticles with different molecules. For covalent attachment, the nanoparticle surface has to show free functional groups, such as carboxylic or amine residues.

\section{Evaluation:}

1. In-vitro Evaluation: No standardized evaluation technique is available for evaluation of CDDS because an ideal in vitro model should posses the in vivo conditions of GIT such as $\mathrm{pH}$, volume, stirring, bacteria, enzymes, enzyme activity and other components of food. Generally these conditions are influenced by the diet and physical stress and these factors make it difficult to design a slandered in vitro model. In vitro model used for CDDS are:

a. In vitro Dissolution Test: Dissolution of controlledrelease formulations used for colon-specific drug delivery are usually complex, and the dissolution methods described in the USP cannot wholly mimic in vivo conditions such as those relating to $\mathrm{pH}$, bacterial environment and mixing forces. Dissolution tests relating to CDDS may be carried out using the conventional basket method. Parallel dissolution studies in different buffers may be undertaken to characterize the behavior of formulations at different $\mathrm{pH}$ levels.

Dissolution tests of a colon- specific formulation in various media simulating $\mathrm{pH}$ conditions and times likely to be encountered at various locations in the gastrointestinal tract. The media chosen were, for example, $\mathrm{pH} 1.2$ to simulate gastric fluid, $\mathrm{pH} 6.8$ to simulate the jejunal region of the small intestine, and $\mathrm{pH} 7.2$ to simulate the ileal segment. Entericcoated capsules for CDDS have been investigated in a gradient dissolution study in three buffers. Invitro test for intactness of coatings and carriers in simulated conditions of stomach and intestine Drug release study in $0.1 \mathrm{~N} \mathrm{HCl}$ for 2 hours (mean gastric emptying time) Drug release study in phosphate buffer for 3 hours (mean small intestine transit time).

b. In vitro Enzymatic Test: For this, there are 2 tests:

i. Incubate carrier drug system in fermenter containing suitable medium for bacteria (Streptococcus faccium or B.ovatus) amount of drug released at different time intervals determined.

ii. Drug release study is done in buffer medium containing enzymes (enzyme pectinase, dextranase), or rat or guinea pig or rabbit cecal contents. The amount of drug released in particular time is determined, which is directly proportional to the rate of degradation of polymer carrier. 
2. In-vivo Evaluation: A number of animals such as dogs, guinea pigs, rats and pigs are used to evaluate the delivery of drug to colon because they resemble the anatomic and physiological conditions as well as the microflora of human GIT. While choosing a model for testing a CDDS, relative model for the colonic diseases should also be considered. Eg. Guinea pigs are commonly used for experimental IBD model. The distribution of azoreductase and glucouronidase activity in the GIT of rat and rabbit is fairly comparable to that in the human. For rapid evaluation of CDDS a novel model has been proposed. In this model the human fetal bowel is transplanted into a subcutaneous tullel on the back of thymic nude mice, which vascularizes within 4 weeks, matures and becomes capable of developing of mucosal immune system from the host.

3. Clinical Evaluation Absorption of drugs from the colon is monitored by colonoscopy and intubation. Currently gamma scintigraphy and high frequency capsules are the most preferred techniques employed to evaluate colon drug delivery systems.

- High Frequency Capsule: Smooth plastic capsule containing small latex balloon, drug and radiotracer taken orally. Triggering system is high frequency generator. Release of drug \& radiotracer triggered by an impulse, the release is monitored in different parts of GIT by radiological localization. It checks the absorption properties of drug in colon.

\section{Gammascintigraphy: By means of} gammascintigraphic imaging, information can be obtained regarding time of arrival of a colonspecific drug delivery system in the colon, times of transit through the stomach and small intestine, and disintegration. Information about the spreading or dispersion of a formulation and the site at which release from it takes place can also be obtained. Gammascintigraphic studies can also provide information about regional permeability in the colon. Information about gastrointestinal transit and the release behaviour of dosage forms can be obtained by combining pharmacokinetic studies and gammascintigraphic studies
CONCLUSION: The colonic region of the GIT has become an increasingly important site for drug delivery and absorption. CDDS offers considerable therapeutic benefits to patients in terms of both local and systemic treatment. Colon specificity is more likely to be achieved with systems that utilize natural materials that are degraded by colonic bacterial enzymes.

Considering the sophistication of colon-specific drug delivery systems, and the uncertainty of current dissolution methods in establishing possible in-vitro/invivo correlation, challenges remain for pharmaceutical scientists to develop and validate a dissolution method that incorporates the physiological features of the colon, and yet can be used routinely in an industry setting for the evaluation of CDDS.

\section{REFERENCES}

1. Akala EO, Elekwachi O, Chase V, Johnson H, Marjorie L, Scott K. Organic Redox Initiated Polymerization Process for the Fabrication of Hydro Gel for Colon Specific Drug Delivery. Drug Dev Ind Pharm.,2003; 29:375-386.

2. Luck M and Crabb. J. US20006074689 (2000).

3. Watts PJ and Illum L: US20016200602 (2001).

4. Yang H, Nguye, VA, Dong, LC and WongPS. L.:US6008187 (1999).

5. Dolan TF, Humphrey MJ, Nichols DJ, Philip AK, Dubey RK, Pathak K. Optimizing Delivery of Furbiprofen to the Colon using a Targeted Prodrug Approach. J Pharm Pharmacol, US20006106864 (2000), 2008; 60:607-613.

6. Vandamme Th F and Chaumeil J C. The Use of Polysaccharides to Target drugs to the Colon, CarboPoly, 48, 2002:219-31.

7. Sarasija $\mathrm{S}$ and Hota A. Colon Specific Drug Delivery Systems, Ind J Pharm Sci., 2002; 62(1):1-8.

8. Macfarlane GT and Cummings JH. The Colonic Flora, Fermentation and Large Bowel Digestive Function. In Phillips SF, Pemberton JH, Shorter RG. The Large Intenstine: Physiology, Pathophysiology and Disease. New York: Raven press, 1991: 51.

9. Thomas P and Rhodes J, Absorption of Delayed-release Prednisolone in Ulcerative Colitis and Crohn's Disease, Int J Pharm, 37, 1985: 757-61.

10. Tomlin J and Read NW. The Relation between Bacterial Degradation of Viscous Polysaccharides and Stool Output in Human Beings, Brit J Nutr., 60, 1988, 476.

11. Philip Anil, Betty Philip. Colon Drug Delivery System: A Review on Primary and Novel Approch. Oman Medical Journal, 2010; 25(2).

12. Krishnaiah YSR, Styanarayana S. Colon- Specific Drug Delivery Systems. In Jain NK, Advances in Controlled and Novel Drug Delivery,CBS Publishers and Distributors, New Delhi. 2000: 89119.

13. Bussemer T, Otto, Bodmeier IR. Pulsatile Drug-Delivery Systems. Crit. Rev. There. Drug Carrier System. 2003, 18: 433-458.

14. Chan RP, Pope DJ, Gilbett AP, Sneta PJ, Baron JH and Bennardjones, JF. Studies of Two Novel Sulphasalazine Analogs I.P. Salazide and Balsalazide. Digestive Diseases Sciences. 1983; 28: 609-716. 
15. Chavan, MS, Sant, VP and Nagarsenker MS. Azo-containing Urethane Analogues for Colonic Drug Delivery: Synthesis, Characterization and In-vitro Evaluation. Journal of Pharmacy Pharmacology. 2001; 53: 895-900.

16. Hita, V, Singh R and Jain SK. Colonic Targeting of Metronidazole using Azo Aromatic Polymers, Development and Characterization. Drug delivery, 1997; 4: 19-22.

17. McLeod AD, Friend DR and Toma NT. Glucocorticoid-Dextran Conjugates As Potential Prodrugs for Colon Specific DeliveryHydrolysis in Rat Gastrointestinal Tract Contents. J Pharm Sci. 1994; 83(9): 1284-1288.

18. Encyclopedia of Pharmaceutical Technology Volume 2.

19. Colonic Drug Delivery: Prodrug Approach Pharmaceutical Research, Vol. 18, No. 5, 2001.
20. Modified-Release Solid Formulations for Colonic Delivery Recent Patents on Drug Delivery \& Formulation 2007, 1: 53-63.

21. Pharmaceutical approaches to colon targeted drug delivery systems JPPS, 2003; 6(1):33-66.

22. Platform Technologies for Colon Targeted Drug Delivery System: A Review Article Journal of Pharmacy Research 2010, 3(3): 543-547.

23. Primary and Novel Approaches for Colon Targeted Drug Delivery - A Review http://www.arjournals.org/ijdd.html

24. www.drug delivery technology.com

25. Advances in controlled drug delivery system- N.K. Jain.

How to cite this article:

Reddy RBD, Malleswari K, Prasad G and Pavani G: Colon Targeted Drug Delivery System: A Review. Int J Pharm Sci Res. 2013 ; 4(1); 1-13. 